\title{
BMJ Open Effect of consumption of animal milk compared to infant formula for non- breastfed/mixed-fed infants 6-11 months of age: a systematic review (protocol)
}

\author{
Aamer Imdad, ${ }^{1}$ Julie Melissa Ehrlich (10 , ${ }^{2}$ Joseph Catania, ${ }^{2}$ Emily Tanner-Smith, ${ }^{3}$ \\ Abigail Smith, ${ }^{4}$ Olivia Tsistinas, ${ }^{4}$ Zulfiqar Ahmed Bhutta ${ }^{5}$
}

To cite: Imdad A, Ehrlich JM, Catania J, et al. Effect of consumption of animal milk compared to infant formula for non-breastfed/ mixed-fed infants 6-11 months of age: a systematic review (protocol). BMJ Open 2021;11:e046370. doi:10.1136/ bmjopen-2020-046370

- Prepublication history and additional materials for this paper is available online. To view these files, please visit the journal online (http://dx.doi. org/10.1136/bmjopen-2020046370).

Received 02 November 2020 Revised 22 December 2020 Accepted 06 January 2021

Check for updates

(C) Author(s) (or their employer(s)) 2021. Re-use permitted under CC BY-NC. No commercial re-use. See rights and permissions. Published by BMJ.

For numbered affiliations see end of article.

Correspondence to

Dr Aamer Imdad;

imdada@upstate.edu

\section{ABSTRACT}

Introduction Prevalence rates of breastfeeding remain low even though the World Health Organization (WHO) and the American Academy of Pediatrics recommend exclusive breast feeding for the first 6 months of life in combination with appropriate complementary feeding beyond six 6 months of age. There have been several studies that address the implication of drinking animal milk and/or infant formula on children's health and development when breast feeding is not offered during the first year of life. Vast improvements have been made in infant formula design, which may increase its benefits compared with animal's milk. The objective of this review is therefore to synthesise the most recent evidence on the effects of the consumption of animal milk compared with infant formula in non-breastfed or mixed breastfed infants aged 6-11 months.

Methods and analysis We will conduct a systematic review and meta-analysis of studies that assessed the effect of animal milk compared with formula or mixed-fed (breastmilk and formula) on infants aged 6-11 months. The primary outcomes of interest include anaemia, gastrointestinal blood loss, weight for age, height for age and weight for height. We will include randomised and non-randomised studies with a control group. We will use the Cochrane risk of bias tools to assess the risk of bias. We will use meta-analysis to pool findings if the identified studies are conceptually homogenous and data are available from more than one study. We will assess the overall quality of evidence using the Grading of Recommendations Assessment, Development, and Evaluation approach.

Ethics and dissemination This is a systematic review, so no patients will be directly involved in the design or development of this study. The findings from this systematic review will be disseminated to relevant patient populations and caregivers and will guide the WHO's recommendations on formula consumption versus animal milk in infants aged 6-11 months.

Trial registration number CRD42020210925.

\section{INTRODUCTION}

The World Health Organization (WHO) and the American Academy of Pediatrics recommend exclusive breastfeeding for the first 6
Strengths and limitations of this study

- We will search several databases for relevant literature and will included randomised and nonrandomised studies.

- We will assess risk-of-bias for each outcome and use the Grading of Recommendations Assessment, Development, and Evaluation to assess the overall quality of evidence.

- We will conduct subgroup analysis for age group (7 months vs 9 months), country, type of feeding (nonbreastfed versus mixed fed) and type of animal milk.

- We will conduct meta-analyses if data are available from more than one study, and if there is clinical and methodological homogeneity in the included studies.

- All the included studies may not report the data for infants $6-11$ months of age which will be a limitation of the study.

months of life and continuation of breastfeeding beyond 6 months in combination with appropriate complementary feedings for up to 2 years or beyond. ${ }^{12}$ However, prevalence rates of breastfeeding still remain low in many contexts, with many infants still being given formula milk or cow's milk before or after 6 months of age. ${ }^{34}$ Use of cow's milk in infancy has been associated with gastrointestinal blood loss, iron deficiency anaemia and increased solute load for kidneys. ${ }^{5-8}$ The composition of cow's milk is vastly different from that of breastmilk and infant formula, which is manufactured to mimic breastmilk. ${ }^{9}$ Cow's milk has a high amount of protein $(3.4 \mathrm{~g} / 100 \mathrm{~mL}$ vs $0.7-1.4100 \mathrm{~mL}$ in breastmilk), ${ }^{10}$ and the composition of the protein is different-50\%-80\% of breastmilk is comprised of whey protein versus only $18 \%$ for cow's milk. ${ }^{9}$ Ingesting a large amount of protein in the first year of life has been associated with childhood obesity. ${ }^{6}{ }^{9}$ Unmodified goat's milk also contains high amounts 
of protein and mineral content and low folate content. Goat's milk does not appear to have any clear nutritional advantage over cow's milk and is not less allergenic. ${ }^{11}$ There have been several studies that have examined the implications of drinking animal milk and/or infant formula on children's health and development. ${ }^{5} 7$ 12-17 The past two decades have also yielded vast improvements in the design of infant formulas, which may increase the benefits of infant formula over animal milk. ${ }^{18}$ The objective of the proposed review is, therefore, to synthesise the most recent research on the effects of the consumption of animal milk compared with infant formula in nonbreastfed or mixed breastfed infants aged 6-11 months of age.

\section{Objective}

For non-breastfed or mixed-fed (breastmilk and formula) infants 6-11 months of age, is the consumption of animal milk compared with infant formula, associated with beneficial or adverse outcomes for health and development?

\section{METHODS AND ANALYSIS}

\section{Study type}

We will include individual and cluster randomised trials. We will also include quasi-randomised experimental design studies, defined as those that attempted to randomly allocate participants to conditions but the method of randomisation was not truly random. ${ }^{19}$ For instance, an example of a quasi-randomised design is an experimental study where the randomisation was attempted but the methods of sequence generation were inadequate such as randomisation based on date of birth of participants or date of enrolment in the study etc. We will also include cohort studies, defined as a study on a group of participants based on certain criteria (eg, geography, age and so on) but none of the participants have the outcome of interest at the time of recruitment and develop outcomes over time in relation to exposure versus no exposure to a risk factor. A cohort study could be prospective or retrospective. ${ }^{19}$ We will exclude case control studies, case series, case reports and crosssectional studies. All analyses will be conducted and reported separately for randomised and non-randomised studies.

\section{Population}

We will include studies that included apparently healthy infants 6-11 months of age who were non-breastfed or mixed fed (breastmilk and formula) irrespective of gestational age and birth weight. We will include studies that started animal milk feeding between 4 months and 11 months of age. If a study includes participants who began intervention before 4 months of age but includes participants older than 4 months of age as well, we will consider that study eligible if more than half $(50 \%)$ of the study participants meet the inclusion criteria (ie, 4-11 months of age). We will include studies where participants are diagnosed with HIV or have had HIV exposure. We will exclude studies if participants are diagnosed with AIDS. We will exclude studies that focused on breastfed infants only without other types of milk provided. We will exclude studies with participants who have chronic diseases such as bronchopulmonary dysplasia, genetic disorders, aerodigestive problems or congenital anomalies.

\section{Intervention}

We will include studies that assessed the effect of animal milk in infants $6-11$ months of age. The animal milk could be cow milk, goat milk or camel milk. We will include studies in which animal milk was the main milk drink as defined by study authors or more than $50 \%$ of the infant's milk intake was animal milk. We will include studies irrespective if the animal milk was boiled or not boiled, pasteurised or unpasteurised, or if the animal milk was full-fat, reduced fat or skim milk. We will exclude studies that used plant-based milk such as rice milk, pea milk, coconut milk or almond milk.

\section{Comparison}

The comparison group in the included studies will be formula feeding or mixed feeding (ie, breast feeding and formula feeding). We will include studies irrespective of the type of formula used; namely, this could include cow's milk-based formula, partially or extensively hydrolysed formula or plant-based formulas such as soy formula. The Federal Food, Drug, and Cosmetic Act defines infant formula as 'a food which purports to be or is represented for special dietary use solely as a food for infants by reason of its simulation of human milk or its suitability as a complete or partial substitute for human milk'. ${ }^{20}$

\section{Outcomes}

\section{Primary outcomes}

- Anaemia (dichotomous outcome, as defined by authors) at 7 months, 9 months, 12 months and the longest follow-up.

- Gastrointestinal blood loss (dichotomous outcome based on stool occult testing) at 7 months, 9 months, 12 months and the longest follow-up.

- Weight for age ( $\mathrm{kg}$ or $\mathrm{Z}$ scores) at 7 months, 9 months, 12 months and the longest follow-up.

- Height for age (cm or Z scores) at 7 months, 9 months, 12 months and the longest follow-up.

- Weight for height $\mathrm{Z}$ score at 7 months, 9 months, 12 months and the longest follow-up.

\section{Secondary outcomes}

- Iron deficiency anaemia (dichotomous outcome) at 7 months, 9 months, 12 months and the longest follow-up.

- Blood iron level (continuous outcome) at 7 months, 9 months, 12 months and the longest follow-up.

- Blood ferritin level (continuous outcome) at 7 months, 9 months, 12 months and the longest follow-up. 
- Haemoglobin concentration in the stool (continuous outcome) at 7 months, 9 months, 12 months and the longest follow-up.

- Haemoglobin concentration in the serum (continuous outcome) at 7 months, 9 months, 12 months and the longest follow-up.

- Fatty acid status (continuous outcome) at 7 months, 9 months, 12 months and longest follow-up.

- Gut health: Diarrhoea (>3 loose stools per day) (dichotomous outcome) at 7 months, 9 months, 12 months and the longest follow-up.

- Gut health: Constipation $(<3$ bowel movements per week) (dichotomous outcome) at 7 months, 9 months, 12 months and longest follow-up.

- Pneumonia (as defined by authors) (dichotomous outcome) at 7 months, 9 months, 12 months and the longest follow-up.

- Allergy (IgE-mediated and non-IgE-mediated and mixed) (dichotomous outcome) at 7 months, 9 months, 12 months and the longest follow-up.

We will include both immunoglobulin $\mathrm{E}$ (IgE)mediated, non-IgE-mediated and mixed milk allergies. IgE-mediated allergies can be identified with a skin prick test or blood test for specific IgE and symptoms typically arise immediately after ingesting the milk product. NonIgE-mediated allergies will not be identified with a skin prick test or blood test and symptoms typically arise 48 hours after ingestion of milk product. ${ }^{21}$ Non-IgE-mediated allergies can be identified using allergen elimination and standard challenge procedure in which the allergen is removed from the diet for up to 2 weeks and then reintroduced to determine if symptoms are elicited. ${ }^{22}$

- Obesity (dichotomous outcome) at 1 year and longest follow-up.

Obesity is defined as a body mass index (BMI) at or above the 95th percentile for children and teens of the same age and sex. ${ }^{23}$ It can also be defined as BMI for age $\mathrm{Z}$ scores $>3$ for children under 5 years of age.

- Overweight (dichotomous outcome) at 1 year and the longest follow-up.

Overweight is defined as a BMI at or above the 85th percentile and below the 95th percentile for children and teens of the same age and sex. ${ }^{23}$ It can also be defined as BMI for age $\mathrm{Z}$ scores $>2$ for children under 5 years of age.

- Neurodevelopmental outcomes (continuous outcome) at 1 year and the longest follow-up.

The term neurodevelopment is a composite term that refers to cognitive, neurological and/or sensory outcomes. This may include intellectual disability as measured on the Mental Developmental Index of the Bayley Scales of Infant Development, gross motor delay measured on Gross Motor Function Classification System and so on.

If a study does not report outcomes at 7 months but instead reports outcomes at 6 months or 8 months, it will be included with outcomes at 7 months. Similarly, if a study does not report outcomes at 9 months but instead reports outcomes at 10 months, it will be included with outcomes at 9 months. If a study does not report outcomes at 12 months, but instead reports those outcomes at 11 , 13 or 14 months, it will be included with outcomes at 12 months.

\section{Literature search}

We will conduct systematic electronic searches on multiple databases, including PubMed, EMBASE, the Cochrane Central Register for Controlled Trials, Web of Science, CINHAL, Scopus, WHO Global Index Medicus. There will be no restrictions applied to the searches based on outcomes, study design, publication status, publication date or language. A proposed search strategy for PubMed is shown in online supplemental appendix 1 . We will search ClinicalTrials.gov to identify ongoing studies. We will also search the websites of relevant international agencies, such as the WHO (including WHO's Reproductive Health Library), UNICEF, Global Alliance for Improved Nutrition, International Food Policy Research Institute, International Initiative for Impact Evaluation (3ie), Nutrition International, World Bank, USAID and affiliates (eg, FANTA, SPRING) and the World Food Program. The reference sections of previously published reviews and the latest published studies will also be searched for potentially eligible studies.

\section{Data extraction and synthesis}

\section{Selection of studies}

Searches from all the databases will be combined in bibliographic software (EndNote) and duplicates will be removed. Two authors (AI and JC or AI and JME) will first screen the titles to identify potentially eligible studies; any studies retained at this step will proceed to the second stage of screening for a full-text review; finally, any studies deemed eligible during the full-text review will proceed to the third step of full data extraction. Any conflict will be resolved by discussion and with the help of the senior author on the team if needed. We will use coding software (Covidence) to conduct the screening. ${ }^{24}$ If a study is only available in abstract form, we will write to authors to obtain details on methods and results. If a study is available in a language other than English, we will attempt to have the translation completed using the local resources. If a study was published in more than one report (multiple publications), we will count those multiple reports as a single study and extract information from all the available reports as needed.

\section{Data extraction}

A data extraction sheet will be designed and piloted for the extraction of information from the selected studies (online supplemental file 2). Two authors (JME and JC) will independently extract the data and compare their findings. Any conflict will be resolved by discussion and with the help of the senior author on the team if needed. We will extract the information on type of study (randomised control trial, quasirandomised experimental 
design or cohort study) study site, study year, study population (age, nutritional status, per cent women), methods for data collection and analysis, intervention (dose, duration, frequency), age of outcome assessment, comparison, outcomes, whether the results were adjusted for confounders, what confounders were considered (socioeconomic status, maternal employment, maternal age, maternal level of education, age-appropriate complementary feeding) and risk of bias (online supplemental document).

\section{Assessment of risk of bias in included studies}

Study risk of bias will be assessed using the Cochrane risk of bias tool (ROB 2.0 $)^{25}$ for randomised controlled trials and using the Cochrane risk of bias in non-randomised studies (ROBINS-I) tool for non-randomised studies of interventions. ${ }^{26}$ Two review authors will independently evaluate and agree on the risk of bias for the individual studies for an outcome. Any disagreements will be resolved by discussion and if no agreement can be made, the senior review author will be consulted. We will assess the effect of assignment to intervention (the intentionto-treat effect) by addressing five domains of signalling questions including: bias arising from the randomisation process, bias due to deviations from intended interventions, bias due to missing outcome data, bias in measurement of the outcome and bias in selection of the reported result. Each domain will get a ranking of low risk of bias, some concerns of bias or high risk of bias. We will include quotes from the study for each signalling question as evidence for our ranking decision. The overall risk of bias will be determined based on the worst ranking for individual domains. For example, if only one domain is ranked 'some concerns', then the overall risk of bias will be 'some concerns'.

\section{DATA SYNTHESIS}

We will develop a Preferred Reporting Items for Systematic Reviews and Meta-Analyses (PRISMA) flow diagram based on the search strategy and eligibility assessment to show the flow of included and excluded studies. ${ }^{27}$ We will analyse the randomised and non-randomised studies separately. We will report findings from all included studies in a narrative synthesis and will also conduct meta-analyses to synthesise evidence across studies quantitatively. Metaanalyses will be conducted when data are available from more than one study, and when there is clinical and methodological homogeneity in the included studies. Dichotomous outcomes will be measured using relative risk effect sizes and will be reported with their corresponding $95 \%$ CIs. Continuous outcomes will be measured using mean difference effect sizes and will also be reported along with their 95\% CIs. We will use the generic inverse variance weighting method for meta-analysis. We will use the random effects model for meta-analysis given that there might be heterogeneity in effects due to variability in the study populations and interventions used. We will use RevMan ${ }^{28}$ and Stata $^{29}$ software for the statistical analysis.

In the case of morbidity outcomes, we will combine all available data whenever possible if outcomes are measured in different ways. For example, we will include all types of diarrhoea (mild, moderate and severe) as a dichotomous value (yes/no) if participants had greater than 3 instances of loose stools per day. We will include occurrence of anaemia, gastrointestinal blood loss based on stool occult testing, iron deficiency anaemia, pneumonia and allergies (non-IgE, IgE-mediated) throughout the study as dichotomous values (yes/no). For dichotomous outcomes, we will extract the total number of participants in each group and the number of participants experiencing an event. We will pool the dichotomous outcomes in a meta-analysis to obtain a summary estimate in the form of relative risk and report it with its corresponding 95\% CI. We will include haemoglobin concentration in stool, weight for age, height for age and weight for height as continuous values. For continuous outcomes, we will pool the data to obtain a pool mean difference and report it with its $95 \%$ CI. In the event that data are reported in different units (eg, few studies report weight in $\mathrm{kg}$ and the others report in $\mathrm{Z}$ scores), we will use a standardised mean difference effect size and report it with its $95 \%$ CI.

\section{Unit of analysis issues}

Multiple-arm trial

Studies with multiple treatment arms will be included if eligible. For multiple-arm trials, we will include data in a way that the only difference between the groups is use of animal milk.

\section{Cluster trials}

Cluster assignment trials will be analysed together with individual randomised trials. We will use the cluster adjusted values; if the trial results are not adjusted for cluster design, we will adjust the result by methods given in the Cochrane handbook for systematic reviews. ${ }^{19}$

\section{STUDIES WITH MISSING DATA}

We will document attrition during data extraction. If the authors report the missing data and report the imputations for the missing data, we will use the latter. If data are missing for some cases, or if reasons for dropout are not reported, we will contact the trial authors to request the full data. In the event that a study does not report the SD for the continuous outcome, we will write to authors to request the data. If the SD data are not available from authors, we will use SD from a similar study that has similar study population. We will prefer to use the final values of a continuous outcome for a given follow-up. If the final values are not available but the difference between the end and the start of the study, we will write to authors to request the final values. If the final values are not available, we will use the difference or rate of change. 


\section{Assessment of heterogeneity}

Assessment of heterogeneity in meta-analysis determines how different the included studies are from each other. Clinical heterogeneity refers to differences in patient population or treatment whereas methodological heterogeneity refers to differences in how the study was designed. Statistical heterogeneity refers to large differences in the outcome (outside of chance) that may be due to clinical or methodological heterogeneity. ${ }^{30}$ Statistical heterogeneity will be assessed using Tau, ${ }^{2} \chi^{2}$ and $\mathrm{I}^{2}$ statistics. We will assess statistical heterogeneity by visual inspection of forest plots, by performing the $\chi^{2}$ test (assessing the $\mathrm{p}$ value) and by calculating the Tau ${ }^{2}$ and $\mathrm{I}^{2}$ statistic. Statistical heterogeneity will be considered substantial if the $\mathrm{p}$ value is less than $0.10, \mathrm{I}^{2}$ value exceeds $50 \%$ and inspection of forest plots show substantial variability in the effect of the intervention. We will perform subgroup analysis to determine the reasons for any identified statistical heterogeneity.

\section{Assessment of reporting bias}

Small study and publication bias will be assessed using funnel plots and regression tests for funnel plot asymmetry when the meta-analysis includes at least ten studies.

\section{Subgroup analysis and investigation of heterogeneity}

- Age group: 7 months vs 9 months.

If a study does not begin intervention at 7 months but instead at 6 months or 8 months, it will be included in the 7 months age subgroup. Similarly, if a study does not begin intervention at 9 months but instead at 10 months, it will be included with outcomes at 9 months.

- Country: Low-income and middle-income country versus high-income country.

- Type of feeding: non-breastfeeding versus mixed feeding.

- Type of animal milk: Cow, goat, buffalo, camel, sheep. We will test the subgroups difference by using $\chi^{2}$ test.

\section{SENSITIVITY ANALYSIS}

- Studies with a high overall risk of bias excluded.

- Random versus fixed effect meta-analysis model.

\section{Rating of overall quality of evidence}

We will assess the overall quality of evidence for the effect of the intervention on each primary outcome using the Grading of Recommendations Assessment, Development, and Evaluation (GRADE) using the software GradePro. ${ }^{31}$ The GRADE approach entails identifying and assessing many different features that affect the certainty of evidence in the review, including type of study design, statistical heterogeneity, directness of evidence, within-study risk of bias, risk of publication bias and precision of effect estimates. ${ }^{32}$ We will consider evidence from randomised trials as high quality and downgrade the evidence one level for serious limitations (table 1).

\begin{tabular}{|c|c|c|c|}
\hline Study design & $\begin{array}{l}\text { Quality of } \\
\text { evidence }\end{array}$ & Lower if & Higher if \\
\hline \multirow{12}{*}{$\begin{array}{l}\text { Randomised } \\
\text { trial }\end{array}$} & \multirow[t]{12}{*}{ High } & Risk of bias & Large effect \\
\hline & & -1 Serious & +1 Serious \\
\hline & & -2 Very serious & +2 Very serious \\
\hline & & Inconsistency & Dose response \\
\hline & & -1 Serious & $\begin{array}{l}+1 \text { Evidence of a } \\
\text { gradient }\end{array}$ \\
\hline & & -2 Very serious & $\begin{array}{l}\text { All plausible } \\
\text { confounding }\end{array}$ \\
\hline & & Indirectness & $\begin{array}{l}+1 \text { Would reduce } \\
\text { a demonstrated } \\
\text { effect or }\end{array}$ \\
\hline & & -1 Serious & $\begin{array}{l}+1 \text { Would suggest } \\
\text { a spurious effect } \\
\text { when results } \\
\text { show no effect }\end{array}$ \\
\hline & & -2 Very serious & \\
\hline & & Imprecision & \\
\hline & & -1 Serious & \\
\hline & & -2 Very serious & \\
\hline \multirow{3}{*}{$\begin{array}{l}\text { Quasi } \\
\text { randomised } \\
\text { trial }\end{array}$} & Moderate & Publication bias & \\
\hline & Low & -1 Serious & \\
\hline & Very low & -2 Very serious & \\
\hline
\end{tabular}

We will rate the overall quality of the body of evidence as very low (we have very little confidence in the effect estimate), low (we have limited confidence in the effect estimate), moderate (we have moderate confidence in the effect estimate; the true effect is likely close to the estimate of the effect) or high (we have high confidence that the true effect lies close to that of the estimate of the effect). We will present the results of the GRADE assessment in the form of a summary of findings tables for the primary outcomes.

\section{AMENDMENTS}

We will do the literature searches, screening of titles, selection of studies, data extraction and analysis according to a priori plan described in this protocol. If we do any additional analysis or change any of the a priori strategies, we will clearly describe that in the methods section.

\section{Patient and public involvement}

No patient or public involvement was considered in preparation of this protocol.

\section{Consent for publication}

Authors give consent for publication of this review.

\section{Availability of data and materials}

We will keep all the data available for review by the editors and peer reviewers and will provide raw data for the general public on request. 


\section{ETHICS AND DISSEMINATION}

This is a systematic review and there will be no direct contact with the patients. The findings from this systematic review will be disseminated to relevant patient populations and caregivers as they will be used to guide recommendations through the $\mathrm{WHO}$ on whether the use of infant formula is better than animal's milk in infants aged 6-11 months.

\section{Author affiliations}

${ }^{1}$ Department of Pediatrics; Division of Pediatric Gastroenterology, Hepatology and Nutrition, SUNY Upstate Medical University, Syracuse, NY, USA

${ }^{2}$ School of Medicine, SUNY Upstate Medical University, Syracuse, NY, USA

${ }^{3}$ College of Education, University of Oregon, Eugene, Oregon, USA

${ }^{4}$ Health Sciences Library, SUNY Upstate Medical University, Syracuse, NY, USA

${ }^{5}$ Institute for Global Health and Development, The Aga Khan University, Karachi, Sindh, Pakistan

Acknowledgements We acknowledge Laurence Grummer-Strawn (Department of Nutrition and Food Safety World Health Organisation), Helen Crawley (First Steps Nutrition, UK), Kathryn Dewey (University of California, Davis, USA) and Nandi Siegfried and Chessa Lutter for their support in development of this protocol.

Contributors Al, JME and JC wrote the first draft of the manuscript. AS and OT developed the search strategy. ET-S provided the support for methodology. ZAB provided the support for the content being evaluated in the review. All authors edited and reviewed the manuscript.

Funding This study is supported by funding from World Health Organisation. Grant number: 2020/1050195-0.

Competing interests None declared.

Patient consent for publication Not required.

Provenance and peer review Not commisioned; peer reviewed for ethical and funding approval prior to submission.

Supplemental material This content has been supplied by the author(s). It has not been vetted by BMJ Publishing Group Limited (BMJ) and may not have been peer-reviewed. Any opinions or recommendations discussed are solely those of the author(s) and are not endorsed by BMJ. BMJ disclaims all liability and responsibility arising from any reliance placed on the content. Where the content includes any translated material, BMJ does not warrant the accuracy and reliability of the translations (including but not limited to local regulations, clinical guidelines, terminology, drug names and drug dosages), and is not responsible for any error and/or omissions arising from translation and adaptation or otherwise.

Open access This is an open access article distributed in accordance with the Creative Commons Attribution Non Commercial (CC BY-NC 4.0) license, which permits others to distribute, remix, adapt, build upon this work non-commercially, and license their derivative works on different terms, provided the original work is properly cited, appropriate credit is given, any changes made indicated, and the use is non-commercial. See: http://creativecommons.org/licenses/by-nc/4.0/.

\section{ORCID iD}

Julie Melissa Ehrlich http://orcid.org/0000-0001-8636-2640

\section{REFERENCES}

1 PAHO. Guiding principles for complementary feeding of the breastfed child. Washington, DC: Pan American Health Organization, World Health Organization, 2003.

2 Section on B. breastfeeding and the use of human milk. Pediatrics 2012;129:e827-41.

3 Victora CG, Bahl R, Barros AJD, et al. Breastfeeding in the 21st century: epidemiology, mechanisms, and lifelong effect. Lancet 2016;387:475-90.

4 Nguyen PH, Avula R, Headey D, et al. Progress and inequalities in infant and young child feeding practices in India between 2006 and 2016. Matern Child Nutr 2018;14:e12663.
5 Fomon SJ, Ziegler EE, Nelson SE, et al. Cow milk feeding in infancy: gastrointestinal blood loss and iron nutritional status. J Pediatr 1981;98:540-5.

6 Thorsdottir I, Thorisdottir AV. Whole cow's milk in early life. Nestle Nutr Workshop Ser Pediatr Program 2011;67:29-40.

7 Dewey KG, Cohen RJ, Rollins NC. Informal Working group on feeding of Nonbreastfed $\mathrm{C}$. who technical background paper: feeding of nonbreastfed children from 6 to 24 months of age in developing countries. Food Nutr Bull 2004;25:377-402.

8 Dewey K. Guiding principles for feeding Non-breastfed children 6-24 months of age. World Health organization, 2005.

9 Martin CR, Ling P-R, Blackburn GL. Review of infant feeding: key features of breast milk and infant formula. Nutrients 2016;8 doi:10.3390/nu8050279

10 Michaelsen KF, Greer FR. Protein needs early in life and long-term health. Am J Clin Nutr 2014;99:718S-22.

11 Turck D. Cow's milk and goat's milk. World Rev Nutr Diet 2013;108:56-62.

12 Bramhagen AC, Axelsson I. Iron status of children in southern Sweden: effects of cow's milk and follow-on formula. Acta Paediatr 1999;88:1333-7.

13 Male C, Persson LA, Freeman V, et al. Prevalence of iron deficiency in 12-mo-old infants from 11 European areas and influence of dietary factors on iron status (Euro-Growth study). Acta Paediatr 2001;90:492-8.

14 Michaelsen KF, Milman N, Samuelson G. A longitudinal study of iron status in healthy Danish infants: effects of early iron status, growth velocity and dietary factors. Acta Paediatr 1995;84:1035-44.

15 Thorisdottir AV, Ramel A, Palsson Gl, et al. Iron status of one-yearolds and association with breast milk, cow's milk or formula in late infancy. Eur J Nutr 2013;52:1661-8.

16 Thorsdottir I, Gunnarsson BS, Atladottir H, et al. Iron status at 12 months of age - effects of body size, growth and diet in a population with high birth weight. Eur J Clin Nutr 2003;57:505-13.

17 Ziegler EE, Fomon SJ, Nelson SE, et al. Cow milk feeding in infancy: further observations on blood loss from the gastrointestinal tract. $J$ Pediatr 1990;116:11-18.

18 Ahern GJ, Hennessy AA, Ryan CA, et al. Advances in infant formula science. Annu Rev Food Sci Technol 2019;10:75-102.

19 Higgins JPT TJ, Chandler J, Cumpston M. Cochrane handbook for systematic reviews of interventions version 6.0, 2019. www.training cochrane.org/handbook2019

20 The federal food, drug, and cosmetic act. Available: https://www. fda.gov/regulatory-information/laws-enforced-fda/federal-food-drugand-cosmetic-act-fdc-act [Accessed 15 Sep 2020].

21 Walsh J, Meyer R, Shah N, et al. Differentiating milk allergy (IgE and non-IgE mediated) from lactose intolerance: understanding the underlying mechanisms and presentations. Br J Gen Pract 2016;66:e609-11.

22 Koletzko S, Niggemann B, Arato A, et al. Diagnostic approach and management of cow's-milk protein allergy in infants and children: ESPGHAN Gi Committee practical guidelines. J Pediatr Gastroenterol Nutr 2012;55:221-9.

23 Defining childhood obesity BMI for children and teens, 2018. Available: https://www.cdc.gov/obesity/childhood/defining.html\#: : text=Overweight\%20is\%20defined\%20as\%20a,the\%20same\% 20age\%20and\%20sex [Accessed 22 Jul 2020].

24 Covidence systematic review software, veritas health innovation, Melbourne, Australia. Available: www.covidence.org

25 Sterne JAC, Savović J, Page MJ, et al. RoB 2: a revised tool for assessing risk of bias in randomised trials. BMJ 2019;366:14898.

26 Sterne JA, Hernán MA, Reeves BC, et al. ROBINS-I: a tool for assessing risk of bias in non-randomised studies of interventions. BMJ 2016;355:i4919.

27 Moher D, Liberati A, Tetzlaff J, et al. Preferred reporting items for systematic reviews and meta-analyses: the PRISMA statement. $J$ Clin Epidemiol 2009;62:1006-12.

28 The Cochrane Collaboration. Version 5.3. Copenhagen: The Nordic Cochrane Centre, 2014

29 StataCorp LLC. Stata statistical software: release 16. College Station, 2019.

30 Melsen WG, Bootsma MCJ, Rovers MM, et al. The effects of clinical and statistical heterogeneity on the predictive values of results from meta-analyses. Clin Microbiol Infect 2014;20:123-9.

31 Evidence Prime, Inc. GRADEpro GDT: GRADEpro guideline development tool [Software]. McMaster University, 2020. Available: gradepro.org

32 Guyatt G, Oxman AD, Akl EA, et al. GRADE guidelines: 1. Introduction-GRADE evidence profiles and summary of findings tables. J Clin Epidemiol 2011;64:383-94. 